\title{
Mitochondrial DNA 8993 (NARP) mutation presenting with a heterogeneous phenotype including 'cerebral palsy'
}

Royal Liverpool Children's Hospital

(Alder Hey), Department of Clinical Genetics

A Fryer

\section{Department of Neurology \\ R Appleton \\ L Rosenbloom}

University Department of Clinical Neurology, Institute of Neurology, London

M G Sweeney

A E Harding

Correspondence to: Dr Alan Fryer, Royal Liverpool University Hospital, PO Box 147 Liverpool L69 3BX.

Accepted 10 August 1994

\author{
Alan Fryer, Richard Appleton, M G Sweeney, Lewis Rosenbloom, A E Harding
}

\begin{abstract}
The mitochondrial DNA (mtDNA) mutation 8993 is an important cause of Leigh's encephalopathy. A family is reported where other affected members have presented with non-specific delayed development or cerebral palsy. The diagnosis should be considered not only in children with Leigh's encephalopathy, but also in those with mild neurological dysfunction (including cerebral palsy) if there is a pigmentary retinopathy or a family history of neurological or ophthalmological disease. There was some correlation in this family between the disease severity and the proportion of mutant mtDNA in the blood. This mutation appears to segregate to high levels of mutant $m t D N A$ rapidly within pedigrees and the mother of a severely affected child has a high risk of having further children with a high proportion of mutant mtDNA and a severe phenotype.

(Arch Dis Child 1994; 71: 419-422)
\end{abstract}

One of the many pathogenic mitochondrial DNA (mtDNA) mutations is a T-G transversion at base pair 8993 in the ATPase subunit 6 gene. It was first described in 1990 in a family with the clinical syndrome referred to as NARP (neurogenic muscle weakness, ataxia, and retinitis pigmentosa). ${ }^{1}$ The index case developed retinitis pigmentosa at 12 years of age and limb and gait ataxia in her thirties; she had one generalised seizure when aged 24 years. She later became demented and was noted to have an axonal sensory neuropathy. Her sister was asymptomatic at the age of $\mathbf{5 2}$ years, but on examination had retinitis pigmentosa and mild proximal muscle weakness. This woman had a healthy son and a daughter who had symptomatic retinitis pigmentosa, proximal muscle weakness, and mild gait ataxia at the age of 29 years. This daughter had a child who was mentally retarded with retinitis pigmentosa and ataxia. The mtDNA 8993 mutation was identified in this family and there was a correlation between the amount of mutant mDNA and the presence and severity of neurological disease in family members.

Subsequently, 20 families have been published with this mutation ${ }^{2-8}$ and another family reported at a conference (P Shillito, D Doyle, D Pike, et al; British Paediatric Neurology Meeting; Birmingham, 1994). These families have shown similar clinical features and marked intrafamilial variability. Tatuch et al first observed that Leigh's syndrome is the most severe phenotype of this mtDNA disease. ${ }^{2}$ This association was confirmed by other workers ${ }^{356}$ and, in retrospect, the most severely affected child described by Holt et al had a relapsing course suggestive of Leigh's encephalopathy. ${ }^{1}$ Santorelli et al showed that 12 of 50 patients with Leigh's encephalopathy, excluding those with cytochrome oxidase or pyruvate dehydrogenase deficiencies, had the NARP mutation. ${ }^{7}$ A second mutation at position 8993 (T-C) has also been associated with Leigh's encephalopathy. ${ }^{9}$

The observed correlation between disease severity and the percentage of mutant mtDNA has been supported by studies in further families and Tatuch et al have produced a graph of the age of onset of symptoms $v$ the percentage heteroplasmy of the mutation. ${ }^{8}$

We have had the opportunity to study a further family with the NARP mutation and in this report we emphasise the clinical manifestations, particularly their variability within the family.

\section{Patients and methods \\ FAMILY REPORT}

The figure shows the family pedigree. The index case was III 1 , who was hypotonic from birth and was initially diagnosed as having cerebral palsy. He was not walking until the age of 3.5 years, when he developed an encephalitic illness after measles and died. Before his death he was noted to have a metabolic acidosis. No neuropathological studies were performed.

Case III 2 , now aged 14 years, had relatively normal early development but was transferred to a special school (for moderate learning difficulties) at 7 years of age. He has poor coordination with bilateral corticospinal tract dysfunction in his legs. He has had slow speech development and is dysarthric. Fundoscopy shows some early peripheral retinal pigmentation. Plasma concentrations of lactate and pyruvate are normal. He has had bilateral herniorrhaphies.

Case III. 3 was noted to be hypotonic with delayed motor milestones at 6 months of age and then developed myoclonic and clonic seizures. His feeding ability deteriorated and he required nasogastric feeds. His growth parameters fell to less than the third centile. He was noted to have periods of hyperventilation, particularly during intercurrent infections. Investigations showed a mild but not constant 


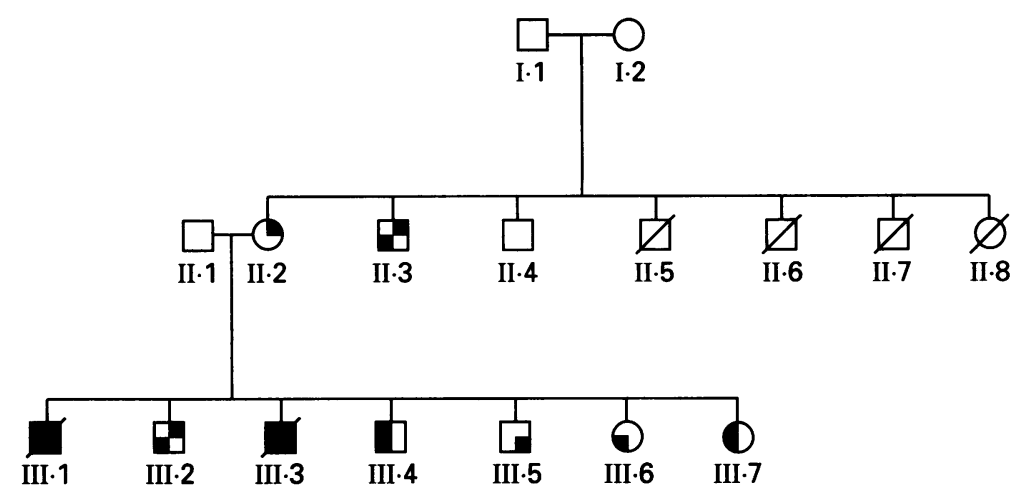

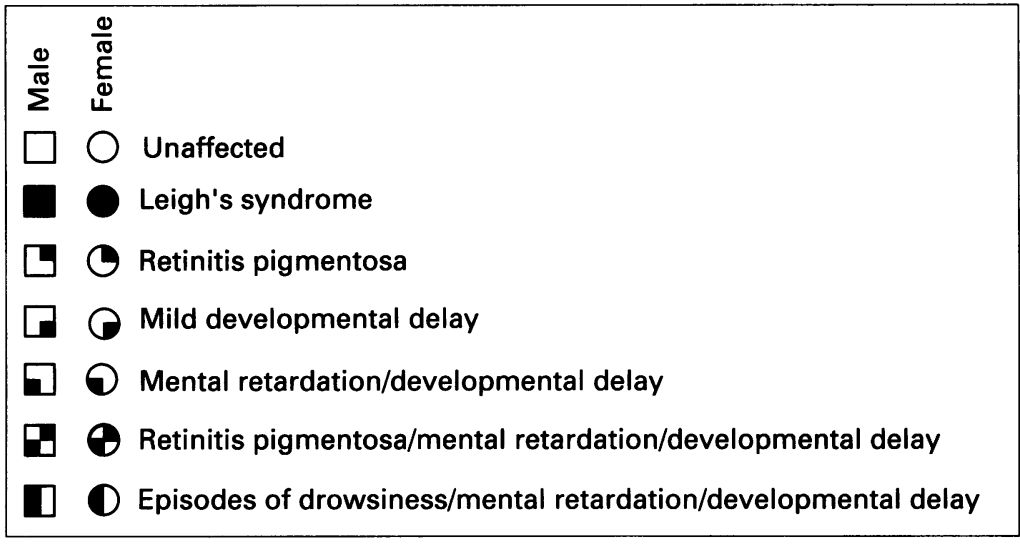

Family pedigree showing phenotypic expression

metabolic acidosis, increased blood concentrations of lactate and pyruvate, and normal white cell pyruvate dehydrogenase and $\alpha$-ketoglutarate dehydrogenase activities. An electroencephalogram showed multifocal abnormal discharges, nerve conduction studies showed a mixed sensory and motor axonal neuropathy, and echocardiography showed evidence of a dilated cardiomyopathy. The electroretinogram response was of low amplitude and poorly defined. Cranial computed tomography two months before death showed cerebral atrophy and areas of low attenuation in both lentiform nuclei. Muscle biopsy (quadriceps) samples showed vacuoles containing fat and glycogen; cytochrome oxidase activity was normal. A putative diagnosis of Leigh's encephalopathy was made. He died at 1 year of age.

Case III.4 appeared to be developing normally until he became hypotonic and drowsy after an upper respiratory tract infection at 15 months of age. Investigations at that time showed a slightly increased blood lactate concentration at $2.47 \mathrm{mmol} / 1$ (normal range $0.63-2.44$ ) and pyruvate $0.13 \mathrm{mmol} / \mathrm{l}$ (normal range $0.03-0.08)$. He recovered from this illness but has subsequently shown delayed development. He did not walk until the age of 2 years. At 9 years of age he has frequent falls, says few words, and cannot speak sentences. He has not lost any previously acquired abilities. Growth parameters (weight, height, and occipitofrontal head circumference) lie between the 10th and 50th centiles. His vision is $6 / 12$ bilaterally and there is no optic atrophy nor abnormal retinal pigmentation. Ocular pursuit is jerky. Hearing is normal but he has delayed speech with a mixed spastic-ataxic dysarthria. Nerve conduction studies showed motor and sensory velocities at the lower range of normal, but the amplitudes of the motor response from extensor digitorum brevis and sural and median nerve action potential amplitudes were reduced, consistent with an axonal sensorimotor neuropathy. Magnetic resonance imaging of his brain is normal. Metabolic studies including plasma lactate and pyruvate, white blood cell lysosomal enzymes, thyroid function, copper, caeruloplasmin, amino (serum and urine) and organic acids (urine), uric acid, serum B-12 and folate, and creatine phosphokinase are normal.

Case III $\cdot 5$, aged 11 years, has had comparatively few problems and is the most mildly affected member of the sibship. He tends to be slightly clumsy and has a slow, immature speech pattern. Neurological and ophthalmological examinations are otherwise normal. His growth parameters and hearing are normal. He attends mainstream school.

Case III.6, aged 6 years, was hypotonic in infancy and by 4.5 years her gross and fine motor skills were at a 2.5 year old level. Her vision is poor ( $6 / 18$ bilaterally), but there is neither optic atrophy nor abnormal retinal pigmentation. Her head circumference is below the 3rd centile and her height and weight are on the 10th centile. Her hearing is normal, but her speech is delayed and dysarthric. There is no ataxia or pyramidal tract dysfunction.

Case III $\cdot 7$, now aged 4.5 years, has been markedly hypotonic from infancy. Her parents recall her being weak and lethargic after a diarrhoeal illness at 6 months of age. She began to walk at 3 years of age with an unsteady gait. At 4 years of age she had a further episode of acute hypotonia and drowsiness lasting 72 hours after an upper respiratory tract infection in association with a metabolic acidosis, slight increase in plasma lactate and an almost threefold increase in plasma pyruvate $(0.19 \mathrm{mmol} / 1$, the normal range being $0.03-0.08$ ). Spontaneous recovery occurred gradually over one week. Her gait is ataxic and tendon reflexes are difficult to elicit. Her visual acuity is reduced bilaterally $(6 / 18)$, but there is no abnormal retinal pigmentation. Her speech is markedly delayed, but her hearing is normal. Her occipitofrontal head circumference is well below the third centile (height and weight between the 3rd and 10th centiles). Magnetic resonance imaging of the brain is normal. Blood concentrations of lactate, pyruvate, and amino acids are normal between episodes; the plasma bicarbonate concentration has been consistently reduced with a mild metabolic acidosis. Motor and sensory conduction velocities are normal; the amplitude of the sural action potential is reduced, which might indicate an early axonal neuropathy.

Case II.2, aged 39 years, was diagnosed as having retinitis pigmentosa at 31 years of age when she presented with poor night vision. She is otherwise clinically normal. Nerve conduction studies have shown evidence of a mild sensory axonal neuropathy.

Case II.3, aged 35 years, is mentally 
handicapped; this was attributed to pertussis immunisation in infancy by the family. He also has retinitis pigmentosa.

Case II $\cdot 4$ is healthy; cases II $\cdot 5-8$ all died in infancy from 'pneumonia'. No information is available on cases $\mathrm{I} \cdot 1$ or $\mathrm{I} \cdot 2$.

\section{METHODS}

DNA was extracted from blood using standard techniques. The mtDNA 8993 (NARP) mutation creates a new restriction site for the enzymes AvaI and HpaII. The relevant region of mtDNA was amplified using primers 8646-8665 (forward) and 9199-9180 (reverse) and the polymerase chain reaction (PCR).

Cycling conditions were $94^{\circ} \mathrm{C}$ for $3 \mathrm{~min}$, then $30 \mathrm{cycles}$ of $94^{\circ} \mathrm{C}(30 \mathrm{sec}), 55^{\circ} \mathrm{C}(30 \mathrm{sec})$, and $72^{\circ} \mathrm{C}$ (30 sec). PCR products were digested with AvaI or HpaII under conditions recommended by the manufacturers (Promega), and separated in a $3 \cdot 2 \%$ agarose gel; in the presence of the 8993 mutation the 553 base pair product is digested into two of 347 and 206 base pairs. For quantification, total genomic DNA was digested with HpaII and separated on a $1.6 \%$ agarose gel. The gel was Southern blotted and hybridised to an mtDNA PCR product spanning 8646-9199 base pairs labelled with ${ }^{32} \mathrm{P}$ by the random primer method (Amersham) and analysed on a Phosphor-Imager (Molecular Dynamics).

\section{Results}

The results of quantitative mtDNA analysis were as follows (proportion of mutant mtDNA): II 2 (78\%); III.2 (88\%); III-4 (86\%); III.5 (87\%); III.6 (93\%); III.7 (93\%). No blood or tissue samples were available from other family members.

\section{Discussion}

The family described here shows exceptionally high penetrance, even for an mtDNA disorder, of the phenotype associated with the NARP mutation, including Leigh's encephalopathy. Until recognition of this syndrome, no connection had been made between the presence of retinitis pigmentosa in the children's mother and uncle and their severe neurological disease. We report this family largely to emphasise the possibility of this underlying mtDNA defect in children with Leigh's encephalopathy, or non-specific delayed development and more mild neurological dysfunction, particularly with pigmentary retinopathy, especially if they have relatives with much milder neurological or ophthalmological disease. Three of the children in our family were initially thought to have cerebral palsy on the basis of their mild and non-progressive neurological dysfunction. Cerebral palsy is a syndrome and not a specific diagnosis and has many different aetiologies; the recurrence of cerebral palsy in siblings or other family members should always raise the suspicion of an underlying, genetically determined disorder. In addition, any child who shows evidence of progressive neurological impairment, with or without acute exacerbations after intercurrent infections, should be considered to have an underlying metabolic or degenerative disease and not 'cerebral palsy'.

In this family, the possibility of a maternally inherited neurological disorder was not seriously considered (by the parents or medical practitioners) until after the birth of the seventh child. One possible explanation for this lies in the marked intrafamilial variation of clinical features; the mother's syndrome was primarily ophthalmological, two of her children (III 1 and III $\cdot 3$ ) showed a rapidly progressive course with early death and two others (III. 2 and III.5) have shown (predominantly) speech and language impairment.

As in other reported families, a muscle biopsy sample did not show the typical features of a mitochondrial encephalomyopathy (ragged red fibres) in the only family member studied. Evidence of metabolic acidosis with increased plasma and lactate concentrations is variable in severely affected children with this disorder, and usually absent in adults with milder phenotypes. Lactate may be increased in the cerebrospinal fluid (although normal in plasma), but this was not measured in our patients.

There was some correlation between disease severity in this family and the proportion of mutant mtDNA in blood in that the mildly affected mother had $78 \%$ mutant mtDNA and all her affected children had levels greater than $85 \%$. Within this sibship, the two most severely affected children available for study had more than $90 \%$ mutant mtDNA. These results are roughly in accord with the findings of Tatuch et al, who concluded that most patients will show early developmental delay where the percentage of mutant mtDNA exceeds $90 \%,{ }^{8}$ whereas milder forms of the disease are associated with $80 \%$ mutant mtDNA; below $75 \%$ retinal dystrophy is likely to be the only feature. Subjects with less than $50 \%$ mutant mtDNA are likely to be asymptomatic.

As appears to be true in other families, ${ }^{7} 10$ the NARP mutation appears to segregate to high levels of mutant mtDNA rapidly within pedigrees. Accordingly, it seems that the mother of a severely affected child has a high risk of having further children with a high proportion of mutant mtDNA and a severe phenotype. The reason for this is unclear, although segregation to almost $100 \%$ mutant mtDNA is a feature of the mtDNA mutations causing Leber's hereditary optic neuropathy. ${ }^{11}$ It is possible that the other members of generation II who died in infancy had the severe form of NARP. Detection of this mutation is important in the diagnosis of Leigh's encephalopathy, or in children with a less specific neurological disorder (including unexplained 'cerebral palsy'), particularly if there is a maternal or family history of neurological or eye disease, or premature death, and obviously has implications in terms of genetic counselling. 
Financial support from the Muscular Dystrophy Group of Great Britain and Northern Ireland is gratefully acknowledged. The authors are grateful to Linda Finnegan for her patience and assistance in the preparation of this manuscript.

1 Holt IJ, Harding AE, Petty RKH, Morgan-Hughes JA. A new mitochondrial disease associated with mitochondr ial DNA heteroplasmy. Am ₹ Hum Genet 1990; 46: 428-33.

2 Tatuch Y, Christodoulou J, Feigenbaum A, et al. Heteroplasmic mtDNA mutation (T-G) at 8993 can cause Leigh disease when the percentage of abnorma mtDNA is high. Am f Hum Genet 1992; 50: 852-8.

3 Shoffner JM, Fernhoff PM, Krawiecki NS, et al. Subacute necrotizing encephalopathy: oxidative phosphorylation defects and the ATPase 6 point mutation. Neurology 1992; 42: 2168-74.

4 Robinson BH, Tatuch YM. Four families with variable presentation of the $8993 \mathrm{mtDNA}$ mutation within the presentation of the $8993 \mathrm{mtDNA}$ mutation within the
ATPase 6 gene [Abstract]. Am $\mathcal{f}$ Hum Genet 1992; 51: ATPa
5 Ciafaloni E, Santorelli FM, Shanske S, et al. Maternally inherited Leigh's syndrome. F Pediatr 1993; 122: 419-22. 6 Sakuta R, Goto Y, Horai S, et al. Mitochondrial DNA mutation and Leigh's syndrome. Ann Neurol 1992; 32: 597-8.

7 Santorelli FM, Shanske S, Macaya A, DeVivo D, DiMauro $\mathrm{S}$. The mutation at nt8993 of mitochondrial DNA is a common cause of Leigh's syndrome. Ann Neurol 1993; 34: 827-34.

8 Tatuch Y, Pagon RA, Vlcek B, Roberts R, Korson M, Robinson BH. The 8993 mtDNA mutation: heteroplasmy and clinical presentation in three families. Eur $\mathcal{J}$ Hum Genet 1994; 2: 35-43.

9 de Vries DD, van Engelen BGM, Gabreels FJM Ruitenbeek W, van Oost BA. A second missense mutation in the mitochondrial ATPase 6 gene in Leigh's syndrome. Ann Neurol 1993; 34: 410-2.

10 Harding AE, Holt IJ, Sweeney MG, Brokington M, Davis MB. Prenatal diagnosis of mitochondrial DNA 8993 T-G disease. Am f Hum Genet 1992; 50: 629-33.

11 Smith KH, Johns DR, Heher KL, Miller NR. Heteroplasmy in Leigh's hereditary optic neuropathy. Arch Ophthalmol 1993; 111: 1486-90. 